\title{
Reconstructing the demographic history of divergence between European river and brook lampreys using Approximate Bayesian Computations
}

Quentin Rougemont, Camille Roux, Samuel Neuenschwander, Jerome Goudet, Sophie Launey, Guillaume Evanno

Inferring the history of isolation and gene flow during species divergence is a central question in evolutionary biology. The European river lamprey (Lampetra fluviatilis) and brook lamprey (L. planeri) show a low reproductive isolation but have highly distinct life histories, the former being parasitic-anadromous and the latter non-parasitic and freshwater resident. Here we used microsatellite data from six replicated population pairs to reconstruct their history of divergence using an approximate Bayesian computation framework combined with a random forest model. In most population pairs, scenarios of divergence with recent isolation were outcompeted by scenarios proposing ongoing gene flow, namely the Secondary Contact (SC) and Isolation with Migration (IM) models. The estimation of demographic parameters under the SC model indicated a time of secondary contact close to the time of speciation, explaining why SC and IM models could not be discriminated. In case of an ancient secondary contact, the historical signal of divergence is lost and neutral markers converge to the same equilibrium as under the less parameterized model allowing ongoing gene flow. Our results imply that models of secondary contacts should be systematically compared to models of divergence with gene flow, and given the difficulty to discriminate among these models; we suggest that genome-wide data are needed to adequately reconstruct divergence history. 
1 Reconstructing the demographic history of divergence between European river and brook lampreys

2 using Approximate Bayesian Computations

3

4 Quentin Rougemont ${ }^{1,2}$, Camille Roux ${ }^{3}$, Samuel Neuenschwander ${ }^{3,4}$, Jérôme Goudet ${ }^{3}$, Sophie Launey ${ }^{1,2}$, 5 Guillaume Evanno ${ }^{1,2}$

6

7 Author for Correspondence: quentinrougemont@orange.fr

8

9

$10{ }^{1}$ Institut National de la Recherche Agronomique, UMR 985 Ecologie et Santé des Ecosystèmes, Rennes, 11 France

${ }^{2}$ Agrocampus Ouest, UMR 985 Ecologie et Santé des Ecosystèmes, Rennes, France

${ }^{3}$ Department of Ecology and Evolution, University of Lausanne, Switzerland 


\section{Abstract}

Inferring the history of isolation and gene flow during species divergence is a central question in evolutionary biology. The European river lamprey (Lampetra fluviatilis) and brook lamprey (L. planeri) show a low reproductive isolation but have highly distinct life histories, the former being parasiticanadromous and the latter non-parasitic and freshwater resident. Here we used microsatellite data

27 from six replicated population pairs to reconstruct their history of divergence using an approximate Bayesian computation framework combined with a random forest model. In most population pairs, scenarios of divergence with recent isolation were outcompeted by scenarios proposing ongoing gene flow, namely the Secondary Contact (SC) and Isolation with Migration (IM) models. The estimation of demographic parameters under the SC model indicated a time of secondary contact close to the time of speciation, explaining why SC and IM models could not be discriminated. In case of an ancient secondary contact, the historical signal of divergence is lost and neutral markers converge to the same equilibrium as under the less parameterized model allowing ongoing gene flow. Our results imply that models of secondary contacts should be systematically compared to models of divergence with gene flow, and given the difficulty to discriminate among these models; we suggest that genome-wide data are needed

37 to adequately reconstruct divergence history. 
41

42

43

44

\section{Introduction}

Understanding the spatio-temporal conditions favouring species emergence is a fundamental question in evolutionary biology. One long standing controversy concerns the geographical setting promoting species divergence (Butlin, Galindo \& Grahame, 2008; Fitzpatrick, Fordyce \& Gavrilets, 2008).

Theory predicts that the accumulation of genetic incompatibilities is rather straightforward under allopatric conditions without gene-flow (Turelli, Barton \& Coyne, 2001; Coyne \& Orr, 2004; Barton \& de Cara, 2009). In contrast, speciation with gene flow theoretically requires (i) strong divergent selection and non-random mating, (ii) high genetic variance and (iii) non-random association of traits under disruptive selection and those involved in assortative mating (Dieckmann \& Doebeli, 1999; Gavrilets, 2003, 2014; Coyne \& Orr, 2004). Importantly, the current geographical distribution of contemporary species may not reflect the initial conditions of divergence as most species may have undergone alternative phases of splits and contacts over historical periods (Hewitt, 1996, 2004, 2011; Bierne et al., 2011). As a result, reconstructing the history of demographic events that have shaped the genetic architecture of present-day populations is of primary importance to understand how speciation operates and infer the role of gene flow during divergence. The accuracy of this reconstruction will depend on an adequate statistical method for demographic inferences, but also on the relevance of the sampling scheme.

Simulation-based methods are helpful for inferences although the tested models often do not entirely reflect the complexity of the usually unknown demographic history of the populations studied (Wakeley, 2008). For instance, several studies using full likelihood approaches implemented in the IM and IMa programs (Hey \& Nielsen, 2004, 2007; Hey, 2010) have compared isolation with migration (IM) models against a model of strict isolation ( $\mathrm{SI}$ ) and revealed a widespread effect of gene flow during divergence (e.g. Pinho, Harris \& Ferrand, 2008; Niemiller, Fitzpatrick \& Miller, 2008; Strasburg \& 
64 Rieseberg, 2008). However, the method makes a number of simplifying assumptions (Strasburg \&

65 Rieseberg, 2010, 2011) and does not allow reconstructing complex scenarios with several parameters, 66 due to computation burden or intractable likelihood computation. Thus the complexity of demographic 67 events may have been missed (Nielsen \& Wakeley, 2001; Hey, 2010). For instance, most of these studies 68 failed to distinguish between primary versus secondary differentiation (i.e. allopatric divergence followed by secondary contacts) hence no general conclusion about the ubiquity of either mechanism during speciation can be drawn yet. Recent advances in coalescent theory (Wakeley, 2008) and Bayesian methods (Tavare et al., 1997; Beaumont, Zhang \& Balding, 2002; Beaumont, 2010; Csilléry et al., 2010) now allow for explicit tests of alternatives and complex models of divergence. In particular, Approximate Bayesian Computation ( $A B C$ ) bypasses the need to compute full likelihoods, as this is not possible or computationally too intensive for complex models with many parameters and large datasets (Beaumont, Zhang \& Balding, 2002). ABC has been used with success to test alternative models of divergence in various taxa and provided useful information on the level of interspecific introgression and complexity of demographic history underlying population divergence (Fagundes et al., 2007; Duvaux et al., 2011; Roux et al., 2013, 2014; Nadachowska-Brzyska et al., 2013; Nater et al., 2015). drawing general conclusions may be complicated with such a reduced sampling scheme. On the other hand, studies of replicated pairs of diverging populations have proven very useful to understand the genetic mechanisms of divergence and speciation by showing that populations can independently evolve similar reproductive barriers in the face of ongoing gene-flow (e.g. Schluter \& McPhail, 1993; Nosil, Crespi \& Sandoval, 2002; Colosimo et al., 2005; Johannesson et al., 2010). Such results were generally interpreted as evidence for parallel adaptation of diverging populations due to the recent action of natural selection. However, alternative scenarios of divergence including secondary contacts 
87 after periods of allopatry have rarely been investigated (Bierne, Gagnaire \& David, 2013; Butlin et al., 88 2014; Welch \& Jiggins, 2014). lineage (jawed vertebrates) approximately 590 million years ago (Hedges et al., 2015). Most lampreys occur as pairs of closely related species reproducing in the same rivers but with very distinct adult life history strategies. Within such pairs, one taxon migrates at sea and becomes parasitic-hematophagous while the other is freshwater-resident, non-parasitic and does not feed at the adult stage (Docker, 2009). Despite a large number of evolutionary and developmental studies in lampreys (Heimberg et al., 2010; Shimeld \& Donoghue, 2012; Smith et al., 2013; Lagadec et al., 2015), there is a high uncertainty about taxonomic relationships among these so called lamprey 'paired' species (Docker, 2009). For instance, the European river lamprey (Lampetra fluviatilis) and brook lamprey (L. planeri) display marked morphological differences at the adult stage: individuals of the anadromous and parasitic river lamprey are on average 2.2 times longer than resident and non-parasitic brook lampreys but adults of both species have been found on the same spawning ground (Lasne, Sabatié \& Evanno, 2010) and this size difference likely forms the most important prezygotic barrier to gene-flow (Beamish \& Neville, 1992; Rougemont et al., 2015). The genetic differentiation between these two taxa is usually low when measured either with allozymes (Schreiber \& Engelhorn, 1998), mtDNA (Espanhol, Almeida \& Alves, 2007; Blank, Jürss \& Bastrop, 2008) or microsatellites markers (Bracken et al., 2015; Rougemont et al., 2015) and these species have been hypothesized to be different ecotypes of a single species (Docker, 2009). Only one study, based on restricted-associated-DNA sequencing of a single population pair, reported a strong differentiation between L. planeri and L. fluviatilis (Mateus et al., 2013). The only large scale phylogeographic study based on mtDNA revealed a very low level of divergence that was hypothesized to result from ongoing gene flow or very recent divergence following postglacial dispersion (Espanhol, Almeida \& Alves, 2007). However, it is known that widespread mtDNA 
111 introgression among sympatric taxa can easily obscure their taxonomic relationships (Shaw, 2002). More

112 recently Bracken et al., (2015) draw similar conclusions of recent divergence following postglacial

113 dispersion based on data from northern European populations. However, phylogeographic approaches

114 do not allow contrasting alternative scenarios of divergence and do not address gene flow following

115 divergence. As a consequence, relatively little is known so far on the history of divergence between $L$.

116 fluviatilis and L. planeri and most conclusions have been related to recent postglacial divergence

117 (Espanhol, Almeida \& Alves, 2007; Bracken et al., 2015) or linked to ecological processes (Salewski,

118 2003). Overall, few studies have used a wide number of pairs of river and brook lamprey connected by

119 gene flow and realistic scenarios of demographic history have never been modelled.

120 Recently Rougemont et al., (2015) studied ten pairs of sympatric and parapatric populations of $L$.

121 fluviatilis and L. planeri and found varying levels of genetic differentiation ranging from strong gene flow

$122\left(F_{\mathrm{ST}}=0.008\right)$ to important genetic differentiation $\left(F_{\mathrm{ST}}=0.189\right)$ depending on population pairs. They

123 concluded that these two species may represent partially reproductively isolated ecotypes, a statement

124 that was consistent with the low degree of reproductive isolation measured in experimental crosses

125 (Hume et al., 2013; Rougemont et al., 2015). However, this pattern of low genetic differentiation

126 observed in several population pairs can be explained by two opposite hypotheses: (i) ongoing

127 divergence with gene flow or (ii) secondary contact after a period of allopatry that did not allow the

128 accumulation of sufficient reproductive barriers, including endogenic barriers. Here we investigated the

129 demographic history of divergence between river and brook lampreys by testing five models of

130 divergence including these two competing scenarios with an $A B C$ approach on microsatellite data

131 obtained in six population pairs of Lampetra.

132 Materials and Methods 

northern France (data from Rougemont et al., 2015 and Fig. 1). Three pairs coexist in sympatry (Aa, Bethune and Oir Rivers) and 2 pairs are not strictly sympatric because a small obstacle occurs on the first river (Hem), while in the second case, populations are located $8 \mathrm{~km}$ apart, on the same stream section (Bresle). The last pair is parapatric (Risle), but with a moderate $F_{\mathrm{ST}}$ value similar to what is observed in sympatric populations (Rougemont et al., 2015). We focused on pairs highly connected by gene flow previously identified in Rougemont et al., study because in disconnected pairs, inferences on the long populations. In parapatric situations L. planeri populations were generally geographically isolated in upper parts of rivers due to anthropogenic barriers to migration with no opportunity for gene flow with speciation process. The sampling included temporal replicates on the Oir (2010, 2011 and 2014), Bresle (2011 and 2014), and Risle rivers (2011 and 2014). A set of 13 microsatellites was used to genotype a total of 727 individuals following the protocol described in Gaigher et al., (2013).

\section{Summary statistics}

Given the lack of genetic differentiation between samples collected in different years in the same river, they were merged together (Rougemont et al., 2015). Similarly, we pooled brook lamprey individuals sampled in upstream and downstream areas on the Aa and Hem river as they displayed no significant genetic differentiation (Table S1). We also pooled river lamprey individuals from the Aa and Hem rivers given the lack of differentiation between these populations (Rougemont et al., 2015). Summary statistics were then computed for each pooled sample. As summary statistics used for comparison between simulated and observed datasets, we computed the average and standard 
157 heterozygosity ( $\mathrm{Ho}$ and $\mathrm{He}$ ), allele size in base pairs, the Garza-Williamson index (GW, Garza \&

158 Williamson, 2001), $G_{S T}(N e i, 1973)$ and $\delta \mu^{2}$ (Goldstein et al., 1995). The GW index (M ratio) aims at

159 assessing reductions in effective population size and is calculated as $M=\frac{A}{R+1}$ with $A$ is the number of

160 alleles for a given loci in each population and $\mathrm{R}$ is the allelic range. Following (Excoffier, Estoup \&

161 Cornuet, 2005) we add 1 to the denominator to avoided dividing by zero in case of a monomorphic

162 sample. Goldstein et al $\delta \mu^{2}$ is a measure of genetic distance developed for microsatellite data defined as

$163 \delta \mu 2=\left(\mu_{1}-\mu_{2}\right)^{2}$ where $\mu_{1}$ and $\mu_{2}$ represents the average number of allelic size differences within

164 populations 1 and populations 2 respectively. Each statistics was computed within populations as well as

165 globally except for the Gst and $\delta \mu^{2}$ which are pairwise statistics. All statistics were computed using $R$

166 scripts ( $R$ Core Team, 2015) available on github at

167 https://github.com/QuentinRougemont/MicrosatDemogInference .

Testing alternatives demographic scenario

\section{ABC coalescent simulations}

For each population pair we used an Approximate Bayesian Computation (Beaumont, Zhang \&

Balding, 2002; Csilléry et al., 2010) framework to statistically compare five alternative models of

demographic history (Fig. 2): (1) the two studied populations derive from a single panmictic gene pool

(PAN); (2) a strict isolation model between sister populations (SI); (3) an isolation with migration model

174 (IM); (4) a model allowing ancient migration but recent isolation (AM) and (5) a model of secondary 175 contact after past isolation (SC). The PAN model assumes a single panmictic population with constant 176 population size. The SI model assumes a strict and instantaneous split of the ancestral population into 177 two daughter populations with constant size and no subsequent gene-flow. The IM model assumes continuous gene-flow between daughter populations after the initial split at constant rate over generations. The AM model assumes gene-flow between the two diverging populations during the first 
generations following the split of the ancestral population. The SC model describes the split of an

181

182

ancestral population in two isolated daughter populations, the two evolving lineages then experience gene flow through a secondary contact starting $T_{S C}$ generations ago. For IM, AM and SC models, the number of migrants was scaled by $M=4 . N_{0} \cdot m$, with $M_{1}$ the number of migrants from $L$. fluviatilis to $L$. planeri and $M_{2}$ the number of migrants from L. planeri to $L$. fluviatilis and $m$ the fraction of the population made of migrants from the other population at each generation. Coalescent simulations were performed using the ms software (Hudson, 2002) assuming an infinite-site model of mutation, in which most parameters are scaled by the effective population size of an arbitrarily chosen reference population $\left(N_{\text {ref }}\right)$ with impact on conclusions drawn by the $A B C$ analysis. Each model was also characterized by a scaled effective population size $\theta: \theta_{\mathrm{A}} / \theta_{\text {Ref }}, \theta_{\mathrm{If}} / \theta_{\text {Ref }}, \theta_{\mathrm{lp}} / \theta_{\text {Ref }}$ where $\theta_{\text {Ref }}=4 N_{\text {Ref }} \mu, \mu$ representing the mutation rate per generation. Patterns of genetic diversity suggested that river lamprey populations display a greater Ne than populations of brook lamprey (Rougemont et al. 2015). Thus, $\theta_{\mathrm{If}}$ was sampled on the interval 0-3 and $\theta_{\mathrm{lp}}$ in the interval 0-max $\left(\theta_{\mathrm{lf}}\right)$. $\theta_{\text {Ref }}$ was set to 1 (i.e. we assumed $N_{\text {Ref }}=1,000$ and $\left.\mu=2.5 \mathrm{e}^{-4}\right)$. The panmictic model was only characterized by the unique effective mutation rate $\theta$ which was also modelled on the interval 0-3. All models (except PAN) also incorporated the scaled time of divergence, $\tau_{\text {split }} / 4 N_{\text {Ref, }}$, where $\tau_{\text {split }}$ is the time measured in number of generations and drawn from a uniform distribution in the interval 0-25. The two parameters $\tau_{\text {iso }}$ (AM model) and $\tau_{\mathrm{sc}}$ (SC model) were computed from uniform distributions defined on the interval $0-\tau_{\text {split. }}$. Since the genetics and ecology of lampreys is poorly known, we chose to include commonly used parameters from the literature (Pinho \& Hey, 2010) after exploring different combinations of uninformative priors following Cornuet, Ravigné \& Estoup, (2010)(Table 1). Binary simulated data from ms were converted into microsatellite data using a stepwise mutation model (SMM) in which probability of changes of the repeat number in each mutation event was modelled by a geometrical parameter $\alpha$ distributed following a uniform prior distribution sampled on the interval $0-0.5$. All computations were run in $R$ 
204 and took into account differences in sample size for each of the thirteen loci. Summary statistics were 205 computed from the transformed microsatellite data. One million simulations composed of the thirteen 206 microsatellite loci were computed under each demographic model. All R code used for ABC computation 207 is available at https://github.com/QuentinRougemont/MicrosatDemogInference .

Model Selection

209 $A B C$ approach

We evaluated the posterior probabilities of each demographic model using an $A B C$ framework implemented in the abc package in R (Csilléry, François \& Blum, 2012). We compared all models at a time by computing posterior probabilities using a feed forward neural network based on a nonlinear conditional heteroscedastic regression in which the model is considered as an additional parameter to be inferred. This procedure allows taking into account correlations of summary statistics and distortion hence reducing the problem of curse of dimensionality (Blum and François 2010). In the rejection step, we retained the $0.02 \%$ simulations closest to the observed summary statistics, which were subsequently weighted by an Epanechnikov kernel that peaks when $S_{o b s}=S_{\text {sim }}$. The regression step was performed using 50 neural networks and 15 hidden layers.

\section{$A B C$ cross-validation}

We performed model checking to compute the classification error rate of the inferred model using pseudo-observed simulated datasets (PODS). We randomly selected 1,000 PODS from one million of simulations computed under each simulated model. We used the same $A B C$ selection procedure as above to estimate the probability of the PODS. Knowing the true model we then computed the type I error rate that corresponds to the risk of excluding the previously inferred scenario when it is the true and the type II error rate that corresponds to the risk of selecting the previously inferred scenario when 
226 it is false following Cornuet, Ravigné \& Estoup, (2010). The most important here was the type II error

227 which, with regards to the selected dataset, corresponds to the risks of erroneously selecting the focal

228 scenario. We validated the accuracy of our procedure by performing the same analysis in pairwise

229 model comparisons of all models of divergence with gene flow (AM, IM, SC) against the model of strict

230 isolation. We also pairwise compared all the models of divergence against the model of panmixia. We

231 again computed type I and type II errors using 1000 PODS taken randomly from the prior distribution

232 and running again the same $A B C$ model selection procedure as above.

\section{Random forest model selection and cross-validations}

In parallel to our $A B C$ based model selection and cross-validation procedure we explored the

ability of a random forest algorithm (Breiman, 2001) to discriminate the different models and to estimate which summary statistics were the most informative. Random forest (RF) is a machine-learning algorithm whose use has recently been advocated for model choice in $A B C$ inference to circumvent curse of dimensionality problems and those linked to the choice of summary statistics (Pudlo et al., 2014). This approach is a non-parametric classification algorithm that uses bootstrapped decision trees to perform classification using a set $(p)$ of defined predictor variables (here the summary statistics). Multiple (i.e. hundreds to thousands) decision trees are grown and merged together and the ensemble makes up the forest (Breiman, 2001). Simulations that are not used in tree building at each bootstrap (the so called out-of-bag simulation $\mathrm{OOB}$ ) are then used to compute the OOB error rate, which provides the dimensionality of the data (Cutler et al., 2007) but also estimating the relative importance of variables (here the summary statistics) through rankings. Variable importance is measured by random permutations of the specified variable in $\mathrm{OOB}$ observations and new predictions are then obtained and 
249 forest is its insensitivity to strong correlations and high noise within data (Pudlo et al., 2014). In addition, 250 the RF analysis has the advantage of being computationally far less extensive the ABC cross validation 251 procedure.

We first constructed 6 random forests (one by river) using the randomForestSRC package in $R$ (Ishwaran et al., 2008; Ishwaran \& Kogalur, 2015) allowing for parallelization and fast computations. We grew 1,000 trees on subsets of 50,000 simulated datasets (5\%) that were used as a training set. Prior analysis using different numbers of trees and training set sizes indicated that the OOB errors reach stationarity using between 500-1,000 trees (see also Fig 4), so we did not grow bigger forests that would have required extensive computations. All summary statistics were included to get an estimation of the importance of each variable. This allowed us to estimate the OOB error rate for each comparison, which is similar to a prior error rate in ABC inference (Pudlo et al., 2014). Ultimately our forest was used as a prediction tool to compute the probability that our observed data belongs to one of the 5 alternatives models.

Parameter estimation and cross-validation

Parameter estimation was performed for the best models using nonlinear regressions. We first used a logit transformation of the parameters on the 2,000 best replicate simulations providing the smallest Euclidian distance $\delta$ (Csilléry, François \& Blum, 2012). We then jointly estimated parameters' posterior probability using the neural network procedure implemented in the abc package. We obtained the best model by weighted nonlinear regressions of the parameters on the summary statistics using 50 feed-forward neural networks and 15 hidden layers. We performed posterior predictive checks for cross-validation in an attempt to check the ability of our parameter estimates to generate data summary statistics close to the observed summary statistics. For each best model, we selected 10,000 samples 
272 simulated 10,000 new datasets by using again ms and custom R scripts. We then again plotted the

273 distance between our observed original values and our new simulations and computed the $p$-value for

274 each statistic as the proportion of values that were larger or smaller than the observed value.

275 Results

276

Population diversity and divergence

277

A total of 6 populations pairs ( 727 individuals in total) were analysed using 13 microsatellites

markers. As already observed (Rougemont et al. 2015), the averaged allelic richness of river lamprey

populations was significantly higher than that of brook lampreys $\left(\operatorname{Ar}_{L f}=3.43, \operatorname{Ar}_{L p}=3.116, p=0.0010\right.$,

Table 2). However, there was no significant difference in expected heterozygosity between river

lamprey $\left(\mathrm{He}_{\mathrm{Lf}}=0.507\right)$ and brook lamprey $\left(\mathrm{He}_{\mathrm{Lp}}=0.46\right) .(p=0.208)$. The global population genetic

differentiation between river and brook lampreys was $F_{\mathrm{ST}}=0.061(99 \% \mathrm{Cl}=0.044-0.079)$ and pairwise $F_{\mathrm{ST}}$

ranged from 0 to 0.192 (Table S1). Genetic differentiation among river lamprey populations was

significantly lower $\left(F_{\mathrm{ST}}=0.002\right)$ than among brook lamprey populations $\left(F_{\mathrm{ST}}=0.109\right)(p=0.003)$. No river

lamprey population differed significantly from the others whereas all brook lamprey populations were

significantly differentiated (Table S1). The pairwise differentiation between river lamprey and brook

lamprey within each river ranged from 0.028 (Oir and Bethune) to 0.091 in the Bresle River and was significant in all cases but the Bethune River (Table S1).

Model comparison and misclassification

The classical $A B C$ model-choice and random forest approaches generally yielded similar results 
294 River, the best supported model by both methods was the IM model. In two cases (Hem and Risle), none 295 of the methods was able to accurately discriminate between the two scenarios (SC and IM model).

296 Finally, in the Oir River the two methods gave incongruent results with the model of panmixia (PAN)

297 being the best supported model under the $A B C$ framework, while the RF failed to distinguish between 298 the IM and SC models. classified in all rivers. The PAN model had very low type II errors across all rivers (Table S2) as welle as low type I errors in pairwise model comparisons (Table S3). Type II errors for SI and AM comparisons were high (Table S2 and Table S3). But this was a minor concern here as these models were not supported by our data. Cross-validations comparing the two most probable models (IM and SC) indicated a high risk of selecting the IM model instead of the SC model in all rivers (averaged type II error $=0.4025$ ). In contrast, the risk of selecting the SC model instead of the IM was low (averaged type II error $=0.057)$.

To gain further insights on the performance of our model selection procedure we tested if a random forest approach could help confirming the validity of the empirically rejected models and distinguishing between the IM and SC models. The RF results confirmed that the models of strict isolation, ancient migration and panmixia were classified with a high accuracy (Table 4, Figure 3 and

311 Figure S1). The overall error rate $(28.79 \%)$ hid very different accuracies depending on the models. 312 Average OOB errors in pairwise analyses of IM versus SC models were as high as $45 \%$ demonstrating that 313 it was generally not possible to correctly classify simulated data in their correct categories (see details in 314 Table 4 and Figure S1). The estimation of variable importance (Figure 3 and Figure S1) indicated that the 315 most informative variables were systematically the mean and variance of $G_{S T}$ and of $\delta \mu^{2}$, generally 
316 followed by the estimators of allelic richness and expected heterozygosity in each population and

317 globally (Figure 3, Figure S1 and Table S4).

Parameters estimation from the best models

We estimated the parameters in each population pair for both the IM and SC models that we failed to accurately distinguish and for the PAN model in the Oir population pair (Table 5, see also Table S6 for untransformed values). The accuracy of posterior parameters estimation varied among population pairs, with the $\mathrm{Aa}$, Hem, Bethune and Risle presenting accurate parameters estimation under both the IM and SC model. In general Ne estimates from the IM model were slightly more accurate than those from the SC model. Under both models we generally observed a reduction of $\mathrm{Ne}$ in both river and brook lampreys as compared to their ancestral populations. Under the IM model the respective median effective population size ( $\mathrm{N} e_{\text {Lf }}$ and $\mathrm{N} e_{\text {Lp }}$ ) of the river lamprey and brook lamprey were on average 1.54 and 5.35 times smaller than the ancestral population. Under the SC model, the (averaged) median effective population sizes were 1.09 and 2.86 times smaller in river lamprey and brook lamprey respectively than their ancestral populations (Table 5). Estimates of $\mathrm{Ne}$ were on average 2.96 times larger in river lampreys than in brook lampreys under the IM model. Under the SC model estimates of Ne were 2.54 times larger in river lampreys than in brook lampreys. Under both the IM and SC models we also noted a slight tendency towards higher migration from river lamprey to brook lamprey with a small deviation from the 1:1 ratio: averaged $m$ ratio $=2.14$ and 1.22 under $\mathrm{IM}$ and SC respectively but 95\% Cl's overlapped in 3 out 5 cases (Table 5).. Estimates of divergence time and timing of secondary contact (SC only) yielded variable results and were not always accurately estimated (Table 5). Estimates from the Aa and Hem population pairs were the most accurate under both scenarios. Overall estimates

337 of split times were congruent under the SC model but revealed that populations would have come into 338 secondary contact for a long period with the averaged time of secondary contact representing more 
339 than one third of the time since divergence. Finally, simulations of the Oir population pair were

340 summarized by a single parameter (the effective mutation rate of a panmictic population) that was

341 estimated with high accuracy (Table 5).

Posterior predictive checks

We performed posterior predictive checks in order to assess the ability of the models to

accurately reproduce summary statistics close to our observed statistics based on 10,000 simulated datasets drawn from the posterior distribution and computed the robustness of our inference. Under both the IM and SC models we consistently found some statistics that differed significantly from our observed data, indicating us that the best proposed models can't reproduce exactly the observed

datasets. Genomic variances in heterozygosity and in allelic number are respectively underestimated and overestimated by the best estimated models. In some cases the mean Garza-Williamson index was not accurately reproduced and underestimated (Table S5). Similarly, in three cases, the variance in $F_{\text {ST }}$ that the variance in Allelic allelic richness and the Garza Williamson index were not accurately reproduced by our data (Table S5). These departures between simulations and observations are the results of complex features in the real genomic history of lampreys that our models did not capture. For instance, the proposed models do not allow temporal variation in migration rates or partial linkage to molecular targets of natural selection with various effects, from positive to balancing selection.

\section{Discussion}

Our goal was to test whether we could discriminate alternative scenarios of divergence between river and brook lampreys using a set of microsatellite markers and an $A B C$ approach. We were able to reject the models of strict isolation and of ancient migration. For one population pair (Oir), the model of 
362 discriminate divergence with ongoing gene flow from a model of allopatric divergence followed by

363 secondary contact.

364 Difficulty in distinguishing between ongoing migration and secondary contact

In spite of the availability of large amount of genetic data and computer resources, few studies

have explicitly tested alternative models of divergence (e.g. Ross-Ibarra, Tenaillon \& Gaut, 2009; Duvaux

et al., 2011; Roux et al., 2013, 2014; Butlin et al., 2014). While populations may diverge (and eventually

become reproductively isolated) under various demographic scenarios, our results indicate that distinguishing between primary differentiation (divergence with gene-flow) versus allopatric divergence followed by secondary contact remains difficult when using a limited number of neutral markers, even with advanced computational tools. Indeed, $A B C$ as well as RF cross-validation clearly showed that the two models were wrongly classified almost half the time. The SC model tended to display a larger proportion of simulations wrongly classified into the IM model, a result that can be explained by the higher complexity of this model that displays one supplementary parameter and is inherently more difficult to infer. In contrast, even though the OOB error rate was high in the IM model, it tended to display fewer simulations wrongly classified into the SC model. Given the inherent difficulty to correctly classify the SC model even when it is true, our support for this model in some cases seems conservative and may suggest that it could be the true model under which lampreys have diverged. is in accordance with theoretical expectations from Bierne, Gagnaire \& David, (2013). Using a simple modelling approach they showed how genetic environmental associations at neutral markers such as microsatellites can quickly be lost after secondary contacts and then reach migration/drift equilibrium together with a pattern of isolation by distance, which is the pattern observed in the studied lamprey populations (see Rougemont et al., 2015). This was indeed expected under the relatively high mutation 
David, (2013) applied their model to the well-studied freshwater/marine stickleback system (e.g.

387 Colosimo et al., 2005; Hohenlohe et al., 2010; Hohenlohe, Catchen \& Cresko, 2012), which shares

388 several characteristics with the lamprey system: a single nearly panmictic marine population and small and potentially independent freshwater populations. The application to the stickleback model showed that introgression proceeded independently between the different streams and was strongly asymmetric from the migratory to the resident populations, which is similar to the pattern we observed here (Table 5) under both the isolation with migration model and the secondary contact model. Although we cannot accurately infer the symmetry of migration, the migration appeared slightly higher from river lamprey to brook lamprey in some rivers (Table 5). allopatric divergence is short and secondary contacts are very long, the SC model converges to the same signal as that obtained under the IM model. The same difficulty is expected when comparing a model of ancient migration in which migration lasts a short amount of time and is followed by a long period of divergence without gene flow to a model of strict isolation. Accordingly, these models could not be clearly distinguished in our cross-validations (e.g. Table 4).

401

The failure to reject panmixia in the Oir River can also be explained in the light of Bierne, Gagnaire \& David, (2013) conclusions. It could be linked to the low genetic divergence observed $\left(F_{S T}=\right.$ 0.028) especially given the small number of markers we used, but this pattern of nearly panmixia can 404 also be attributed to a stronger introgression in this system than in all other investigated streams. In this river the mean size of river lampreys ( $225 \mathrm{~mm}, n=134)$ was much smaller than the size observed in other sites (mean $=303 \mathrm{~mm}, n=389$ ). Assuming that size difference is the most important cause of reproductive isolation (Beamish \& Neville, 1992) a smaller size difference may facilitate mating of the 
409

410

412

413

414

415

416

417

418

419

420

421

422

423

424

425

426

427

428

429

430

431

432

neutral markers are necessarily difficult as this pattern may be explained by strong gene flow after an isolation period as well as by a very early stage of ongoing divergence.

Demographic parameter estimations and new insights on lamprey history

We assumed a mutation rate of $2.5 \mathrm{e}^{-4}$, in accordance with what is observed in several fish species (Shimoda et al., 1999; Steinberg et al., 2002; Yue, David \& Orban, 2006) and other vertebrates (e.g. Nance et al., (2011)). together with Nref $=1,000$ based on prior knowledge of possible population size in lampreys (Rougemont et al., unpublished data) we observed asymmetries in effective populations size between river lamprey (averaged median $=1448 ; 95 \% \mathrm{Cl}=890-2300$ under SC, 1158 and 95\% $\mathrm{Cl}=788-1710$ under $\mathrm{IM}$ ) and brook lamprey (averaged median $=612 ; 95 \% \mathrm{Cl}=356-1090$; and $49495 \% \mathrm{Cl}=$ 318-840 under SC and IM respectively). The greater estimates of effective population size in river lamprey reflect the idea that homing is moderate in this species and population size as well as gene flow are large (Rougemont et al. 2015, Spice et al. 2012) resulting in a situation similar to that observed in the panmictic populations of marine sticklebacks (Hohenlohe et al., 2010; Hohenlohe, Catchen \& Cresko, 2012). We also observed reductions in effective population size of each resident brook lamprey population as compared to the ancestral populations (averaged median $=1428 ; 95 \% \mathrm{Cl}=962-1886$; and median $=1748$ with $95 \% \mathrm{Cl}=1490-2026$ under SC and IM respectively). Such a reduction is expected following the independent river colonization by founding individuals from the resident ecotype (Bierne, Gagnaire \& David, 2013).

Estimates of divergence times suggested that the two ecotypes may have split around 201,000 years ago (95\% Cl:148,000-250,000) under the IM model and around 257,000 years ago (95\% Cl:195,000-344,000) under the SC model (assuming a generation time of 5 years, Potter \& Potter, (1971)). Such estimates are rather similar to what was observed in Dicentrarchus labrax (Tine et al., 2014). Consequently, it seems unlikely that the divergence was initiated rapidly, following the recent 
433 glacial retreats around 10,000-15,000 years ago (Bernatchez \& Wilson, 1998; Aldenhoven et al., 2010) as

434 often proposed to explained ecotypic divergence in various aquatic species (eg. (Schluter \& Nagel, 1995;

435 Espanhol, Almeida \& Alves, 2007; Bracken et al., 2015; Mateus et al., 2016) . Importantly, under the SC

model, the secondary contact would have started around 92,000 years ago, representing more than one

third of the total divergence time between the two species. Such an ancient secondary contact implies

that the genetic signature of historical geographic isolation carried by neutral markers may have been

lost. In these conditions, neutral markers can converge to the same state than the one observed under

primary differentiation explaining again the difficulty of discriminating the two models (Barton \& Hewitt,

1985; Charlesworth, Nordborg \& Charlesworth, 1997, Bierne, Gagnaire \& David, 2013). The SC model

implies the accumulation of some Dobzhansky-Muller incompatibilities in allopatry when the two

ecotypes started to diverge. While both theory (Orr, 1995) and empirical evidence (Moyle \& Nakazato,

2009; Matute et al., 2010; Wang, White \& Payseur, 2015) predict that DMI should accumulate faster

than linearly in time, our result suggest a limited amount of isolation. The period of isolation was

certainly too short to allow for sufficient DMI to accumulate and to develop strong barriers to gene flow.

This would likely explain the low differentiation observed for mtDNA (Espanhol, Almeida \& Alves, 2007;

Blank, Jürss \& Bastrop, 2008; Bracken et al., 2015) and is fully compatible with the observation of viable

F1 hybrids (Hume et al., 2013; Rougemont et al., 2015.

Another question we started to address is the origin of parallel divergence observed between

river lamprey and brook lamprey. The process of recent and independent postglacial divergence with

conceptually simple explanation often used to explain divergence between freshwater resident and 
457 and divergence time estimates appeared older than the onset of glacial retreats. Alternative scenarios

458 involve either multiple independent secondary contacts between populations inhabiting different

459 refugia or secondary contact between anadromous parasitic and freshwater resident populations each

460 having diverged in a different ancestral place. Under this scenario a spatial re-assortment of alleles

461 involved in non-parasitism would allow the recolonization of neighboring rivers (i.e. the transporter

462 hypothesis, (Schluter \& Conte, 2009; Bierne, Gagnaire \& David, 2013; Welch \& Jiggins, 2014).

463 Considering (i) the relatively small scale of investigation and (ii) the nearly panmictic situation in river

464 lamprey; the hypothesis of a spatial re-assortment of ancestral variation by migration between adjacent

465 rivers appears more parsimonious. In this case, the history of divergence would reflect a re-

466 interpretation of the transport hypothesis under a scenario of secondary differentiation rather than

467 primary differentiation. The major conceptual difference is the existence of the brook lamprey

468 background before the recent colonization of rivers. This latter process may have arisen either through

469 hybrid genotypes colonizing rivers or transport of alleles broken up by recombination and at low

470 frequency in the river lamprey background. While the global divergence implies secondary contact, it is

471 debatable whether the spatial re-assortment process constitutes divergence with gene flow or

472 secondary contact hence the difficulty in distinguishing between the two models. Disentangling these

473 scenarios of primary versus secondary differentiation under the transporter hypothesis is challenging

474 and may be addressed by combining genome wide data with historical modelling among multiple pairs

475 of populations. Our results also suggest that the RF method provides a valuable complement to the

476 standard ABC model comparison (Robert et al., 2011; Pudlo et al., 2014; Marin et al., 2014). The two

477 methods provided similar outcomes in terms of model choice and subsequent cross-validations except

478 in one population pair (Oir River). Our ability to distinguish between SC and IM was low in both cases. In

479 line with Pudlo et al., (2014) we find that the RF approach possesses a series of advantages over the ABC

480 approach such as 1) fast model choice procedure with simultaneous cross validation through OOB 
481 computations, and 2) considerable reduction of computational time. Estimating variable importance can

482 be particularly interesting when a large set of variables are used without prior knowledge about the 483 pertinence of the summary statistics used. The choice of summary statistics is an important process in 484 ABC methodology (Csilléry et al., 2010). RF may provide such an objective tool that may be 485 complementary to conventional $A B C$ model choice and cross validation procedure. Note however, that 486 the neural network method provided in the abc package, performed very well and provided similar 487 results to the RF model. To our knowledge, this study is the first to empirically combine $A B C$ and RF for 488 model choice and cross-validation.

\section{Conclusion and perspectives}

Our study shed new light on the demographic processes that have shaped the current genetic makeup of population pairs of European river and brook lampreys. In particular, we were able to reject a scenario of divergence in strict isolation and a scenario of ancient sympatric divergence. The scenario of panmixia was also supported only once and it is thus unlikely to be a generalizable scenario across the species range. However, it was not possible to firmly discriminate the SC or IM models but it is likely that distinguishing between these alternatives scenarios is complicated in cases of ancient secondary contacts, especially when investigations are performed with a limited number of neutral markers. In particular, detecting secondary contact may require sufficiently long allopatric divergence and a time of secondary contact that represents only a small portion of the total divergence time. This study thus the prevalence of rapid parallel speciation Bierne, Gagnaire \& David, (2013). In addition, similar analyses may help understanding the divergence between other lamprey species pairs in which interspecific gene 
505 allowing for heterogeneous migration rate among loci (e.g. Roux et al. 2013, Roux et al. 2014), variation

506 of migration rate in time and variation of effective population size along the genome, may allow fine-

507 tuning demographic investigations and provide further insight onto the prevalence of secondary

508 contacts versus speciation with continuous gene-flow in nature.

\section{Acknowledgments}

510 We wish to thank M. Navascues for providing great insights onto the joint use of $\mathrm{R}$ and $\mathrm{ms}$ for

511 microsatellite data. We are also grateful to Jeffrey-Ross Ibarra and two anonymous referees for their

512 insightful comments on the manuscript. We thank the Genotoul bioinformatics platform Toulouse Midi-

513 Pyrenees for providing computing and storage resources.

\section{References}

515 Aldenhoven JT., Miller MA., Corneli PS., Shapiro MD. 2010. Phylogeography of ninespine sticklebacks

516 (Pungitius pungitius) in North America: glacial refugia and the origins of adaptive traits.

517 Molecular Ecology 19:4061-4076. DOI: 10.1111/j.1365-294X.2010.04801.x.

Barton NH., de Cara MAR. 2009. The evolution of strong reproductive isolation. Evolution; International Journal of Organic Evolution 63:1171-1190. DOI: 10.1111/j.1558-5646.2009.00622.x.

Barton NH., Hewitt GM. 1985. Analysis of Hybrid Zones. Annual Review of Ecology and Systematics 16:113-148. DOI: 10.1146/annurev.es.16.110185.000553.

Beamish RJ., Neville C-EM. 1992. The Importance of Size as an Isolating Mechanism in Lampreys. Copeia 1992:191. DOI: 10.2307/1446551.

Beaumont MA. 2010. Approximate Bayesian Computation in Evolution and Ecology. Annual Review of 162:2025-2035. 
528 Bernatchez L., Wilson CC. 1998. Comparative phylogeography of Nearctic and Palearctic fishes.

529 Molecular Ecology 7:431-452. DOI: 10.1046/j.1365-294x.1998.00319.x.

530 Bierne N., Welch J., Loire E., Bonhomme F., David P. 2011. The coupling hypothesis: why genome scans

531 may fail to map local adaptation genes. Molecular ecology 20:2044-2072. DOI: 10.1111/j.1365-

$532 \quad 294 \times .2011 .05080 . x$

533 Bierne, N., Gagnaire, P.A. \& David, P. 2013. The geography of introgression in a patchy environment and 534 the thorn in the side of ecological speciation. Curr. Zool. 59: 72-86.

535

536

537

Blank M., Jürss K., Bastrop R. 2008. A mitochondrial multigene approach contributing to the systematics of the brook and river lampreys and the phylogenetic position of Eudontomyzon mariae. Canadian Journal of Fisheries and Aquatic Sciences 65:2780-2790. DOI: 10.1139/F08-178.

Bracken FSA., Hoelzel AR., Hume JB., Lucas MC. 2015. Contrasting population genetic structure among freshwater-resident and anadromous lampreys: the role of demographic history, differential

Breiman L. 2001. Random Forests. Machine Learning 45:5-32. DOI: 10.1023/A:1010933404324.

Butlin RK., Saura M., Charrier G., Jackson B., André C., Caballero A., Coyne JA., Galindo J., Grahame JW., Hollander J., Kemppainen P., Martínez-Fernández M., Panova M., Quesada H., Johannesson K., Rolán-Alvarez E. 2014. Parallel evolution of local adaptation and reproductive isolation in the

Butlin RK., Galindo J., Grahame JW. 2008. Sympatric, parapatric or allopatric: the most important way to 550 classify speciation? Philosophical Transactions of the Royal Society B: Biological Sciences 363:2997-3007. DOI: 10.1098/rstb.2008.0076. 
552 Charlesworth B., Nordborg M., Charlesworth D. 1997. The effects of local selection, balanced

553 polymorphism and background selection on equilibrium patterns of genetic diversity in

554 subdivided populations. Genetics Research 70:155-174. DOI: null.

555 Colosimo PF., Hosemann KE., Balabhadra S., Villarreal G., Dickson M., Grimwood J., Schmutz J., Myers

556 RM., Schluter D., Kingsley DM. 2005. Widespread Parallel Evolution in Sticklebacks by Repeated

557 Fixation of Ectodysplasin Alleles. Science 307:1928-1933. DOI: 10.1126/science.1107239.

558 Cornuet J-M., Ravigné V., Estoup A. 2010. Inference on population history and model checking using

559 DNA sequence and microsatellite data with the software DIYABC (v1.0). BMC Bioinformatics

560 11:401. DOI: 10.1186/1471-2105-11-401.

561 Coyne JA., Orr HA. 2004. Speciation. W.H. Freeman.

562 Csilléry K., Blum MGB., Gaggiotti OE., François O. 2010. Approximate Bayesian Computation (ABC) in

563 practice. Trends in Ecology \& Evolution 25:410-418. DOI: 10.1016/j.tree.2010.04.001.

564 Csilléry K., François O., Blum MGB. 2012. abc: an R package for approximate Bayesian computation 565 (ABC). Methods in Ecology and Evolution 3:475-479. DOI: 10.1111/j.2041-210X.2011.00179.x.

566 Cutler DR., Edwards TC., Beard KH., Cutler A., Hess KT., Gibson J., Lawler JJ. 2007. Random forests for $567 \quad$ classification in ecology. Ecology 88:2783-2792.

568 Dieckmann U., Doebeli M. 1999. On the origin of species by sympatric speciation. Nature 400:354-357.

$569 \quad$ DOI: $10.1038 / 22521$

570 Docker MF. 2009. A review of the evolution of nonparasitism in lampreys and an update of the paired $571 \quad$ species concept. :71-114.

572 Duvaux L., Belkhir K., Boulesteix M., Boursot P. 2011. Isolation and gene flow: inferring the speciation 573 history of European house mice. Molecular Ecology 20:5248-5264. DOI: 10.1111/j.1365294X.2011.05343.x. 
575 Espanhol R., Almeida PR., Alves MJ. 2007. Evolutionary history of lamprey paired species Lampetra

576 fluviatilis (L.) and Lampetra planeri (Bloch) as inferred from mitochondrial DNA variation.

577 Molecular ecology 16:1909-1924. DOI: 10.1111/j.1365-294X.2007.03279.x.

578 Excoffier L., Estoup A., Cornuet J-M. 2005. Bayesian Analysis of an Admixture Model With Mutations and

$579 \quad$ Arbitrarily Linked Markers. Genetics 169:1727-1738. DOI: 10.1534/genetics.104.036236.

580 Fagundes NJR., Ray N., Beaumont M., Neuenschwander S., Salzano FM., Bonatto SL., Excoffier L. 2007.

581 Statistical evaluation of alternative models of human evolution. Proceedings of the National

582 Academy of Sciences 104:17614-17619. DOI: 10.1073/pnas.0708280104.

Fitzpatrick BM., Fordyce JA., Gavrilets S. 2008. What, if anything, is sympatric speciation? Journal of evolutionary biology 21:1452-1459. DOI: 10.1111/j.1420-9101.2008.01611.x.

Gaigher A., Launey S., Lasne E., Besnard A-L., Evanno G. 2013. Characterization of thirteen microsatellite markers in river and brook lampreys (Lampetra fluviatilis and L. planeri). Conservation Genetics Resources 5:141-143. DOI: 10.1007/s12686-012-9753-z.

Garza JC., Williamson EG. 2001. Detection of reduction in population size using data from microsatellite loci. Molecular Ecology 10:305-318. DOI: 10.1046/j.1365-294X.2001.01190.x.

Gavrilets S. 2003. Perspective: models of speciation: what have we learned in 40 years? Evolution; international journal of organic evolution 57:2197-2215.

592

Gavrilets S. 2014. Models of Speciation: Where Are We Now? Journal of Heredity 105:743-755. DOI: 10.1093/jhered/esu045.

Goldstein DB., Ruiz Linares A., Cavalli-Sforza LL., Feldman MW. 1995. Genetic absolute dating based on microsatellites and the origin of modern humans. Proceedings of the National Academy of Sciences of the United States of America 92:6723-6727.

Hedges SB., Marin J., Suleski M., Paymer M., Kumar S. 2015. Tree of Life Reveals Clock-Like Speciation and Diversification. Molecular Biology and Evolution 32:835-845. DOI: 10.1093/molbev/msv037. 
599 Heimberg AM., Cowper-Sal·lari R., Sémon M., Donoghue PCJ., Peterson KJ. 2010. microRNAs reveal the 600 interrelationships of hagfish, lampreys, and gnathostomes and the nature of the ancestral $601 \quad$ vertebrate. Proceedings of the National Academy of Sciences 107:19379-19383. DOI:

602 10.1073/pnas.1010350107.

603 604 605 606 607 608 609

Hewitt GM. 1996. Some genetic consequences of ice ages, and their role in divergence and speciation. Biological Journal of the Linnean Society 58:247-276. DOI: 10.1111/j.1095-8312.1996.tb01434.x.

Hewitt GM. 2004. Genetic consequences of climatic oscillations in the Quaternary. Philosophical Transactions of the Royal Society of London. Series B, Biological Sciences 359:183-195; discussion 195. DOI: 10.1098/rstb.2003.1388.

Hewitt GM. 2011. Quaternary phylogeography: the roots of hybrid zones. Genetica 139:617-638. DOI: 10.1007/s10709-011-9547-3.

Hey J. 2010. Isolation with Migration Models for More Than Two Populations. Molecular Biology and Evolution 27:905-920. DOI: 10.1093/molbev/msp296.

Hey J., Nielsen R. 2004. Multilocus Methods for Estimating Population Sizes, Migration Rates and Divergence Time, With Applications to the Divergence of Drosophila pseudoobscura and D. persimilis. Genetics 167:747-760. DOI: 10.1534/genetics.103.024182.

Hey J., Nielsen R. 2007. Integration within the Felsenstein equation for improved Markov chain Monte Carlo methods in population genetics. Proceedings of the National Academy of Sciences 104:2785-2790. DOI: 10.1073/pnas.0611164104.

Hohenlohe PA., Bassham S., Etter PD., Stiffler N., Johnson EA., Cresko WA. 2010. Population Genomics of Parallel Adaptation in Threespine Stickleback using Sequenced RAD Tags. PLoS Genet 6:e1000862. DOI: 10.1371/journal.pgen.1000862. 
621 Hohenlohe PA., Catchen J., Cresko WA. 2012. Population Genomic Analysis of Model and Nonmodel 622 Organisms Using Sequenced RAD Tags. In: Pompanon F, Bonin A eds. Data Production and 623 Analysis in Population Genomics. Methods in Molecular Biology. Humana Press, $235-260$.

624 Hudson RR. 2002. Generating samples under a Wright-Fisher neutral model of genetic variation. 625 Bioinformatics 18:337-338. DOI: 10.1093/bioinformatics/18.2.337.

626 Hume JB., Adams CE., Mable B., Bean C. 2013. Post-zygotic hybrid viability in sympatric species pairs: a

627

628

629

630

631

632

633

634

635

636

637 case study from European lampreys. Biological Journal of the Linnean Society 108:378-383. DOI: 10.1111/j.1095-8312.2012.02007.x.

Ishwaran H., Kogalur UB., Blackstone EH., Lauer MS. 2008. Random survival forests. The Annals of Applied Statistics 2:841-860. DOI: 10.1214/08-AOAS169.

Ishwaran H., Kogalur UB. 2015. randomForestSRC: Random Forests for Survival, Regression and Classification (RF-SRC).

Johannesson K., Panova M., Kemppainen P., André C., Rolán-Alvarez E., Butlin RK. 2010. Repeated evolution of reproductive isolation in a marine snail: unveiling mechanisms of speciation. Philosophical Transactions of the Royal Society of London. Series B, Biological Sciences 365:1735-1747. DOI: 10.1098/rstb.2009.0256.

Lagadec R., Laguerre L., Menuet A., Amara A., Rocancourt C., Péricard P., Godard BG., Celina Rodicio M., Rodriguez-Moldes I., Mayeur H., Rougemont Q., Mazan S., Boutet A. 2015. The ancestral role of nodal signalling in breaking L/R symmetry in the vertebrate forebrain. Nature Communications 6. DOI: $10.1038 /$ ncomms7686.

Lasne E., Sabatié M-R., Evanno G. 2010. Communal spawning of brook and river lampreys (Lampetra planeri and L. fluviatilis) is common in the Oir River (France). Ecology of Freshwater Fish 19:323325. DOI: 10.1111/j.1600-0633.2010.00428.x. 
644 Marin J-M., Pillai NS., Robert CP., Rousseau J. 2014. Relevant statistics for Bayesian model choice. 645 Journal of the Royal Statistical Society: Series B (Statistical Methodology) 76:833-859. DOI:

646 10.1111/rssb.12056.

647

648

649

650

651

652

653

654

655

656

657

658

659

660

661

662

663

664

665

666

Mateus CS., Stange M., Berner D., Roesti M., Quintella BR., Alves MJ., Almeida PR., Salzburger W. 2013. Strong genome-wide divergence between sympatric European river and brook lampreys. Current Biology 23:R649-R650. DOI: 10.1016/j.cub.2013.06.026.

Mateus CS., Almeida PR., Mesquita N., Quintella BR., Alves MJ. 2016. European Lampreys: New Insights on Postglacial Colonization, Gene Flow and Speciation. PLOS ONE 11:e0148107. DOI: 10.1371/journal.pone.0148107.

Matute DR., Butler IA., Turissini DA., Coyne JA. 2010. A test of the snowball theory for the rate of evolution of hybrid incompatibilities. Science (New York, N.Y.) 329:1518-1521. DOI: 10.1126/science.1193440.

Moyle LC., Nakazato T. 2009. Complex Epistasis for Dobzhansky-Muller Hybrid Incompatibility in Solanum. Genetics 181:347-351. DOI: 10.1534/genetics.108.095679.

Nadachowska-Brzyska K., Burri R., Olason PI., Kawakami T., Smeds L., Ellegren H. 2013. Demographic divergence history of pied flycatcher and collared flycatcher inferred from whole-genome resequencing data. PLoS genetics 9:e1003942. DOI: 10.1371/journal.pgen.1003942.

Nance HA., Klimley P., Galván-Magaña F., Martínez-Ortíz J., Marko PB. 2011. Demographic Processes Underlying Subtle Patterns of Population Structure in the Scalloped Hammerhead Shark, Sphyrna lewini. PLoS ONE 6:e21459. DOI: 10.1371/journal.pone.0021459.

Nater A., Greminger MP., Arora N., van Schaik CP., Goossens B., Singleton I., Verschoor EJ., Warren KS., Krützen M. 2015. Reconstructing the demographic history of orang-utans using Approximate Bayesian Computation. Molecular Ecology 24:310-327. DOI: 10.1111/mec.13027. 
667 Nielsen R., Wakeley J. 2001. Distinguishing migration from isolation: a Markov chain Monte Carlo 668 approach. Genetics 158:885-896.

669

670

671

672

673

674

675

676

677

678

679

680

681

682

683

684

685

686

687

688

689

690

Niemiller ML., Fitzpatrick BM., Miller BT. 2008. Recent divergence with gene flow in Tennessee cave salamanders (Plethodontidae: Gyrinophilus) inferred from gene genealogies. Molecular Ecology 17:2258-2275. DOI: 10.1111/j.1365-294X.2008.03750.x.

Nosil P., Crespi BJ., Sandoval CP. 2002. Host-plant adaptation drives the parallel evolution of reproductive isolation. Nature 417:440-443. DOI: 10.1038/417440a.

Orr HA. 1995. The population genetics of speciation: the evolution of hybrid incompatibilities. Genetics 139:1805-1813.

Pinho C., Harris DJ., Ferrand N. 2008. Non-equilibrium estimates of gene flow inferred from nuclear genealogies suggest that Iberian and North African wall lizards (Podarcis spp.) are an assemblage of incipient species. BMC Evolutionary Biology 8:63. DOI: 10.1186/1471-2148-8-63.

Pinho C., Hey J. 2010. Divergence with Gene Flow: Models and Data. Annual Review of Ecology, Evolution, and Systematics 41:215-230. DOI: 10.1146/annurev-ecolsys-102209-144644.

Potter MWH\& IC., Potter IC. 1971. The Biology of Lampreys. Volume 1. London, New York: Academic Press.

Pudlo P., Marin J-M., Estoup A., Cornuet J-M., Gautier M., Robert CP. 2014. ABC model choice via random forests. arXiv:1406.6288 [q-bio, stat].

Robert CP., Cornuet J-M., Marin J-M., Pillai N. 2011. Lack of confidence in ABC model choice. arXiv:1102.4432 [q-bio, stat].

Ross-Ibarra J., Tenaillon M., Gaut BS. 2009. Historical Divergence and Gene Flow in the Genus Zea. Genetics 181:1399-1413. DOI: 10.1534/genetics.108.097238.

Rougemont Q., Gaigher A., Lasne E., Côte J., Coke M., Besnard A-L., Launey S., Evanno G. 2015. Low reproductive isolation and highly variable levels of gene flow reveal limited progress towards 
691

692

693

694

695

696

697

698

699

700

701

702

703

704

705

706

707

708

709

710

711

712

713

714

speciation between European river and brook lampreys. Journal of Evolutionary Biology 28:2248-2263. DOI: 10.1111/jeb.12750.

Roux C., Tsagkogeorga G., Bierne N., Galtier N. 2013. Crossing the species barrier: genomic hotspots of introgression between two highly divergent Ciona intestinalis species. Molecular Biology and Evolution:mst066. DOI: 10.1093/molbev/mst066.

Roux C., Fraïsse C., Castric V., Vekemans X., Pogson GH., Bierne N. 2014. Can we continue to neglect genomic variation in introgression rates when inferring the history of speciation? A case study in a Mytilus hybrid zone. Journal of Evolutionary Biology 27:1662-1675. DOI: 10.1111/jeb.12425.

Salewski V. 2003. Satellite species in lampreys: a worldwide trend for ecological speciation in sympatry? Journal of Fish Biology 63:267-279. DOI: 10.1046/j.1095-8649.2003.00166.x.

Schluter D., Conte GL. 2009. Genetics and ecological speciation. Proceedings of the National Academy of Sciences of the United States of America 106 Suppl 1:9955-9962. DOI: 10.1073/pnas.0901264106.

Schluter D., McPhail JD. 1993. Character displacement and replicate adaptive radiation. Trends in Ecology \& Evolution 8:197-200. DOI: 10.1016/0169-5347(93)90098-A.

Schluter D., Nagel LM. 1995. Parallel Speciation by Natural Selection. The American Naturalist 146:292301.

Schreiber A., Engelhorn R. 1998. Population genetics of a cyclostome species pair, river lamprey (Lampetra fluviatilis L.) and brook lamprey (Lampetra planeri Bloch). Journal of Zoological Systematics and Evolutionary Research 36:85-99. DOI: 10.1111/j.1439-0469.1998.tb00781.x.

Shaw KL. 2002. Conflict between nuclear and mitochondrial DNA phylogenies of a recent species radiation: what mtDNA reveals and conceals about modes of speciation in Hawaiian crickets. Proceedings of the National Academy of Sciences of the United States of America 99:1612216127. DOI: $10.1073 /$ pnas. 242585899. 
715

716

717

Shimeld SM., Donoghue PCJ. 2012. Evolutionary crossroads in developmental biology: cyclostomes (lamprey and hagfish). Development 139:2091-2099. DOI: 10.1242/dev.074716.

Shimoda N., Knapik EW., Ziniti J., Sim C., Yamada E., Kaplan S., Jackson D., de Sauvage F., Jacob H., Fishman MC. 1999. Zebrafish Genetic Map with 2000 Microsatellite Markers. Genomics 58:219232. DOI: $10.1006 /$ geno.1999.5824.

Smith JJ., Kuraku S., Holt C., Sauka-Spengler T., Jiang N., Campbell MS., Yandell MD., Manousaki T., Meyer A., Bloom OE., Morgan JR., Buxbaum JD., Sachidanandam R., Sims C., Garruss AS., Cook M., Krumlauf R., Wiedemann LM., Sower SA., Decatur WA., Hall JA., Amemiya CT., Saha NR., Buckley KM., Rast JP., Das S., Hirano M., McCurley N., Guo P., Rohner N., Tabin CJ., Piccinelli P., Elgar G., Ruffier M., Aken BL., Searle SMJ., Muffato M., Pignatelli M., Herrero J., Jones M., Brown CT., Chung-Davidson Y-W., Nanlohy KG., Libants SV., Yeh C-Y., McCauley DW., Langeland JA., Pancer Z., Fritzsch B., de Jong PJ., Zhu B., Fulton LL., Theising B., Flicek P., Bronner ME., Warren WC., Clifton SW., Wilson RK., Li W. 2013. Sequencing of the sea lamprey (Petromyzon marinus) genome provides insights into vertebrate evolution. Nature Genetics 45:415-421. DOI: 10.1038/ng.2568.

Steinberg EK., Lindner KR., Gallea J., Maxwell A., Meng J., Allendorf FW. 2002. Rates and Patterns of Microsatellite Mutations in Pink Salmon. Molecular Biology and Evolution 19:1198-1202.

Strasburg JL., Rieseberg LH. 2008. Molecular demographic history of the annual sunflowers Helianthus annuus and $\mathrm{H}$. petiolaris--large effective population sizes and rates of long-term gene flow. Evolution; International Journal of Organic Evolution 62:1936-1950. DOI: 10.1111/j.15585646.2008.00415.x.

Strasburg JL., Rieseberg LH. 2010. How Robust Are "Isolation with Migration" Analyses to Violations of the IM Model? A Simulation Study. Molecular Biology and Evolution 27:297-310. DOI: 10.1093/molbev/msp233. 
739 Strasburg JL., Rieseberg LH. 2011. Interpreting the estimated timing of migration events between hybridizing species. Molecular Ecology 20:2353-2366. DOI: 10.1111/j.1365-294X.2011.05048.x.

Tavare S., Balding DJ., Griffiths RC., Donnelly P. 1997. Inferring Coalescence Times from DNA Sequence Data. Genetics 145:505-518.

Tine M., Kuhl H., Gagnaire P-A., Louro B., Desmarais E., Martins RST., Hecht J., Knaust F., Belkhir K., Klages S., Dieterich R., Stueber K., Piferrer F., Guinand B., Bierne N., Volckaert FAM., Bargelloni L., Power DM., Bonhomme F., Canario AVM., Reinhardt R. 2014. European sea bass genome and its variation provide insights into adaptation to euryhalinity and speciation. Nature Communications 5. DOI: 10.1038/ncomms6770. 343. DOI: 10.1016/S0169-5347(01)02177-2.

Wakeley J. 2008. Coalescent Theory: An Introduction. Greenwood Village, Colo: Roberts \& Company Publishers.

Wang RJ., White MA., Payseur BA. 2015. The Pace of Hybrid Incompatibility Evolution in House Mice. Genetics 201:229-242. DOI: 10.1534/genetics.115.179499.

754

755

756

757

758

759 760

Welch JJ., Jiggins CD. 2014a. Standing and flowing: the complex origins of adaptive variation. Molecular Ecology 23:3935-3937. DOI: 10.1111/mec.12859.

Welch JJ., Jiggins CD. 2014b. Standing and flowing: the complex origins of adaptive variation. Molecular Ecology 23:3935-3937. DOI: 10.1111/mec.12859.

Yue GH., David L., Orban L. 2006. Mutation rate and pattern of microsatellites in common carp (Cyprinus carpio L.). Genetica 129:329-331. DOI: 10.1007/s10709-006-0003-8. 
$\theta_{\mathrm{A}}, \theta 1, \theta 2$ = effective mutation rate for the ancestral, river lamprey and brook lamprey populations respectively. $M_{1}, M_{2}, M_{\text {Anc }}=$ Effective migration rate for the ancestral, river lamprey and brook lamprey

764 populations respectively. $\tau=$ divergence time, $\tau_{\text {isol, }} \tau_{s c}$ divergence time under the ancient migration

765 model and time of secondary contact respectively. SI: strict isolation, IM: isolation with migration, AM:

766 ancient migration, PAN: Panmixia, SC: secondary contact model.

\begin{tabular}{|c|c|c|}
\hline Parameters & Models & Prior \\
\hline$\theta_{\mathrm{A}}=4 N_{\mathrm{Anc}} \mu$ & $\mathrm{SI}, \mathrm{IM}, \mathrm{AM}, \mathrm{SC}$ & Uniform [0-3] \\
\hline$\theta_{1}=4 N_{1} \mu$ & $\mathrm{SI}, \mathrm{IM}, \mathrm{AM}, \mathrm{SC}, \mathrm{PAN}$ & Uniform [0-3] \\
\hline$\theta_{2}=4 N_{2} \mu$ & $\mathrm{SI}, \mathrm{IM}, \mathrm{AM}, \mathrm{SC}$ & Uniform [0- $\left.\left(\theta_{1}\right)\right]$ \\
\hline$M_{1}=M_{2}=4 N_{1} m$ & $\mathrm{IM}, \mathrm{SC}$ & Uniform [0-20] \\
\hline$M_{\mathrm{ANC}}=4 N_{1} m$ & $\mathrm{AM}$ & Uniform [0-20] \\
\hline$\tau=4 N_{1} t$ & $\mathrm{SI}, \mathrm{IM}, \mathrm{AM}, \mathrm{SC}$ & Uniform [0-25] \\
\hline$\tau_{\text {isol }}=4 N_{1} t$ & $\mathrm{AM}$ & Uniform [0- $\tau]$ \\
\hline$\tau_{\mathrm{sc}}=4 N_{1} t$ & $\mathrm{SC}$ & Uniform [0- $\tau]$ \\
\hline
\end{tabular}


768 Table 2: Estimates of populations genetic parameters for each pair of river and brook lamprey

769 populations. $\mathrm{N}=$ number of individuals used for $A B C$ analysis $A r=$ Allelic richness, $H e=$ expected

770 heterozygosity, GW= Garza-Williamson Index. Population are classified by increasing order of genetic

771 differentiation.

\begin{tabular}{llllllllllll}
\hline Pop & $\begin{array}{l}\text { River } \\
\text { Name }\end{array}$ & \multirow{N}{*}{$L f$} & $\mathrm{~N} L p$ & $F_{\mathrm{ST}}$ & $\mathrm{Ar} L f$ & $\operatorname{Ar} L p$ & $\mathrm{He} L f$ & $\mathrm{He} L p$ & $\mathrm{GW} L f$ & $\mathrm{GW} L p$ & Delta $\mu^{2}$ \\
\hline OIR & Oir & 104 & 74 & 0.028 & 4.45 & 3.61 & 0.52 & 0.508 & 0.525 & 0.622 & 0.204
\end{tabular}

\begin{tabular}{llllllllllll}
\hline BET & Bethune & 14 & 14 & 0.028 & 3.51 & 3.36 & 0.516 & 0.471 & 0.452 & 0.464 & 0.507 \\
\hline RIS & Risle & 75 & 75 & 0.033 & 3.84 & 3.92 & 0.503 & 0.472 & 0.497 & 0.421 & 0.842 \\
& & & & & & & & & & & \\
\hline HEM & Hem & $30^{*}$ & $65^{\delta}$ & 0.077 & 4.21 & 3.53 & 0.504 & 0.477 & 0.406 & 0.487 & 1.633 \\
\hline AA & Aa & $34^{*}$ & $69^{\delta}$ & 0.084 & 4.21 & 3.76 & 0.514 & 0.522 & 0.406 & 0.505 & 0.915 \\
\hline BRE & Bresle & 93 & 80 & 0.091 & 4.14 & 4.91 & 0.49 & 0.49 & 0.466 & 0.263 & 34.37
\end{tabular}

$772{ }^{*}$ For the $\mathrm{ABC}$ inference, individuals of river lamprey from the $\mathrm{AA}$ and $\mathrm{Hem}\left(F_{\mathrm{ST}}=0\right)$ river were pooled

773 together to obtain a sample size similar to the one of brook lampreys.

$774 \delta$ Brook lamprey samples from the AA and Hem rivers are composed of upstream and downstream

775 samples from the Rougemont et al. (2015) study. 
778 Table 3: ABC classification (posterior probability) and random forest (RF) prediction of each model of

779 speciation in each river.

\begin{tabular}{|c|c|c|c|c|c|c|c|c|c|c|c|c|}
\hline \multirow[b]{3}{*}{ RIVER } & \multicolumn{12}{|c|}{ MODEL } \\
\hline & \multicolumn{2}{|c|}{$\mathrm{SI}$} & \multicolumn{2}{|c|}{$\mathrm{IM}$} & 1 & \multicolumn{2}{|c|}{ AM } & 1 & \multicolumn{2}{|c|}{ SC } & \multicolumn{2}{|c|}{ PAN } \\
\hline & $A B C$ & RF & $A B C$ & $\mathrm{RF}$ & $\begin{array}{l}1 \\
1 \\
1\end{array}$ & $A B C$ & $\mathrm{RF}$ & $\begin{array}{l}T \\
1\end{array}$ & $A B C$ & $\mathrm{RF}$ & $A B C$ & $\mathrm{RF}$ \\
\hline$A A$ & $\begin{array}{l}1 \\
1 \\
1 \\
1\end{array}$ & 0 & 0.27 & 0.39 & $\begin{array}{l}1 \\
1 \\
1 \\
1\end{array}$ & 0.01 & 0.06 & $\begin{array}{l}1 \\
1 \\
1\end{array}$ & 0.72 & 0.54 & 0 & 0 \\
\hline BET & i & 0 & 0.45 & 0.57 & $\begin{array}{l}1 \\
1\end{array}$ & 0 & 0.02 & $\begin{array}{l}1 \\
\vdots\end{array}$ & 0.46 & 0.35 & 0.08 & 0.06 \\
\hline BRE & 0.01 & 0.02 & 0.12 & 0.24 & $\begin{array}{l}1 \\
1 \\
1\end{array}$ & 0.14 & 0.12 & $\begin{array}{l}1 \\
i\end{array}$ & 0.73 & 0.62 & 0 & 0 \\
\hline HEM & $\begin{array}{l}1 \\
1 \\
1\end{array}$ & 0 & 0.42 & 0.53 & $\begin{array}{l}1 \\
1 \\
1\end{array}$ & 0.01 & 0.05 & $\begin{array}{l}1 \\
1 \\
1\end{array}$ & 0.57 & 0.42 & 0 & 0 \\
\hline RIS & i & 0 & 0.46 & 0.47 & 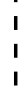 & 0 & 0 & i & 0.54 & 0.52 & 0 & 0 \\
\hline OIR & 1 & 0 & 0.15 & 0.46 & ! & 0 & 0.02 & i & 0.14 & 0.47 & 0.71 & 0.05 \\
\hline Average & 0.00 & 0.00 & 0.31 & 0.44 & I & 0.03 & 0.05 & i & 0.53 & 0.49 & 0.13 & 0.02 \\
\hline
\end{tabular}

780

781 
782 Table 4: random forests out-of-bag confusion matrix and classification error. Data based on 6 random

783 forests, each composed of 1,000 trees based on a trained set of 50,000 simulated predictor variables

784 (summary statistics). The response variable is the demographic model. Proportions of correctly classified

785 demographic models are in bold. The grey italic values represent models with high error rates.

786 Simulation between rivers differed only by the number of individual loci simulated and produced very

787 similar values that were subsequently averaged over each demographic model.

\begin{tabular}{|ccccccc|}
\cline { 2 - 7 } \multicolumn{1}{c|}{} & \multicolumn{5}{c}{ Predicted Model (Averaged over each river) } & $\begin{array}{c}\text { Averaged OOB } \\
\text { error rate }\end{array}$ \\
\hline Observed & AM & I & IM & PAN & SC & \\
\hline AM & $\mathbf{7 8 . 0 \%}$ & $16.7 \%$ & $2.4 \%$ & $0.0 \%$ & $2.9 \%$ & $21.99 \%$ \\
\hline I & $25.2 \%$ & $\mathbf{7 3 . 6 \%}$ & $0.6 \%$ & $0.0 \%$ & $0.6 \%$ & $26.38 \%$ \\
\hline IM & $1.6 \%$ & $0.2 \%$ & $57.2 \%$ & $0.8 \%$ & $40.1 \%$ & $42.76 \%$ \\
\hline PAN & $0.0 \%$ & $0.0 \%$ & $0.2 \%$ & $\mathbf{9 9 . 7 \%}$ & $0.1 \%$ & $0.30 \%$ \\
\hline SC & $2.1 \%$ & $0.3 \%$ & $43.6 \%$ & $0.6 \%$ & $\mathbf{5 3 . 3 \%}$ & $47.12 \%$ \\
\hline
\end{tabular}



population size of the single population (made of both Lf and Lp backgrounds).

\begin{tabular}{|c|c|c|c|c|c|c|c|c|}
\hline River & Model & $\mathrm{Ne}$ Lf & $\mathrm{Ne} \mathbf{L} \mathbf{p}$ & $\begin{array}{l}\mathrm{Ne} \text { Ancestral } \\
\text { population }\end{array}$ & $\begin{array}{c}\text { migration from } \\
\text { Lp to Lf }\end{array}$ & $\begin{array}{l}\text { migration } \\
\text { from Lf to Lp }\end{array}$ & Split time & $\begin{array}{l}\text { Time secondary } \\
\text { contact }\end{array}$ \\
\hline \multirow{3}{*}{ AA } & & median [95HPD] & median [95HPD] & median [95HPD] & median [95HPD] & median [95HPD] & median [95HPD] & median [95HPD] \\
\hline & IM & $1310[930-2020]$ & $410[290-680]$ & $2290[2260-2350]$ & $0.0025[0.0018-0.0032]$ & $0.003[0.0027-0.003]$ & $268000[246000-282000]$ & \\
\hline & SC & $1480[760-2550]$ & $390[270-620]$ & $1850[1600-2110]$ & $0.0022[0.002-0.0025]$ & $0.0032[0.003-0.0033]$ & $191200[168000-230000]$ & $89200[61400-108000]$ \\
\hline \multirow[t]{2}{*}{ BET } & IM & $1620[1260-2350]$ & $940[430-1540]$ & $1650[1500-1780]$ & $0.0029[0.0027-0.0032]$ & $0.0035[0.0033-0.0037]$ & $29800[24200-34200]$ & \\
\hline & SC & $1930[1280-2630]$ & $940[570-1420]$ & $1160[1030-1300]$ & $0.0024[0.002-0.0027]$ & $0.0033[0.0032-0.0038]$ & $322000[265600-396400]$ & $\begin{array}{c}164600[116000- \\
212800]\end{array}$ \\
\hline \multirow[t]{2}{*}{ BRE } & IM & $1020[440-2450]$ & $310[120-790]$ & $1360[440-2370]$ & $0.0004[0.0004-0.0015]$ & $0.002[0.0006-0.0039]$ & $274000[99800-453800]$ & \\
\hline & SC & 1610[910-2720] & $740[220-1500]$ & $1440[320-2620]$ & $0.0017[0.0004-0.0041]$ & $0.0025[0.0007-0.0046]$ & $268000[140800-446400]$ & $20400[3800-124600]$ \\
\hline \multirow[t]{2}{*}{ HEM } & IM & $1000[710-194]$ & $190[130-310]$ & $2600[2480-2700]$ & $0.002[0.0017-0.0027]$ & $0.0042[0.0039-0.0044]$ & $240000[191200-270000]$ & \\
\hline & SC & $860[660-1280]$ & $350[80-880]$ & $1680[1200-1880]$ & $0.0031[0.0028-0.0034]$ & $0.0024[0.002-0.0029]$ & $278000[231400-328800]$ & $99400[85400-110000]$ \\
\hline \multirow[t]{2}{*}{ RIS } & IM & $840[600-1540]$ & $620[620-880]$ & $840[770-930]$ & $0.0021[0.0015-0.003]$ & $0.0025[0.0018-0.0031]$ & $197000[181800-209400]$ & \\
\hline & SC & $1360[840-2320]$ & $640[640-1030]$ & $1010[660-1520]$ & $0.0036[0.0034-0.004]$ & $0.0037[0.0036-0.0038]$ & $226000[168600-320400]$ & $91200[42600-151800]$ \\
\hline \multirow[t]{2}{*}{$\begin{array}{l}\text { Avera } \\
\text { ge }\end{array}$} & IM & $1158[788-1710]$ & $494[318-840]$ & $1748[1490-2026]$ & $0.0020[0.0016-0.0027]$ & $0.003[0.0025-0.0036]$ & $201760[148600-249880]$ & {$[-]$} \\
\hline & SC & $1448[890-2300]$ & $612[356-1090]$ & $1428[962-1886]$ & $0.0026[0.0021-0.0033]$ & $0.003[0.0025-0.0037]$ & $257040[194880-344400]$ & 92960[61840-141440] \\
\hline OIR & PAN* & $2050[1940-2180]$ & & & & & & \\
\hline
\end{tabular}


794 Figure 1: Map of sampling sites across the channel area. River names match those given in Table 2 and

795 Fst values are given for each population pair.
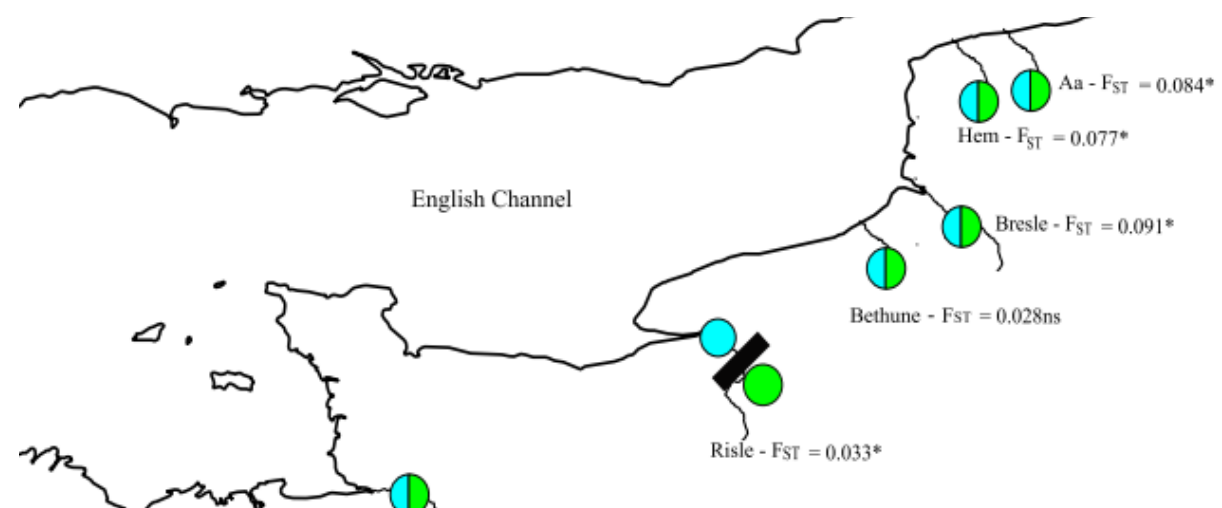

796
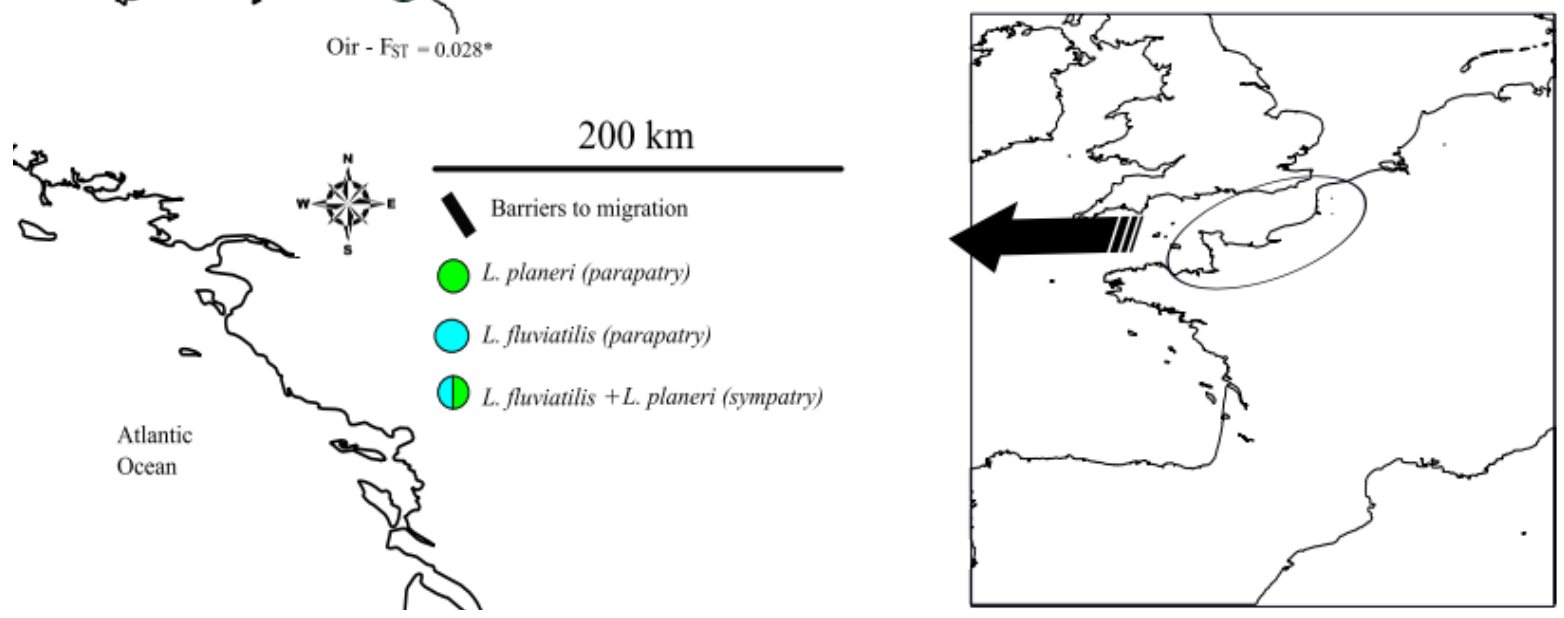
797 Figure 2: Different scenario of divergence between L. planeri and L. fluviatilis.

798 Five models with different parameters are tested and compared. Two null models: Stict Isolation (SI) and 799 Panmixia (PAN). Three models of migration: isolation with constant migration (IM), ancient migration 800 (AM) and secondary contact (SC). The following parameters are shared by all models: $\tau_{\text {div }}$ : number of 801 generations since divergence time. $\theta_{A}, \theta_{L f}, \theta_{L p}$ : effective population size of the ancestral population, of $L$. 802 fluviatilis and L. planeri respectively. $\tau_{\text {isol }}$ is the number of generations since the two ecotypes have 803 stopped exchanging genes. $\tau_{\mathrm{sc}}$ is the number of generations since the two ecotypes have entered into a 804 secondary contact after a period of isolation. $M_{12}$ and $M_{21}$ represent the number of migrants expressed 805 in $4 . \mathrm{Nm}$ units per generation with $m$ the proportion of population made of migrants from the other 806 populations.

Strict Isolation - SI

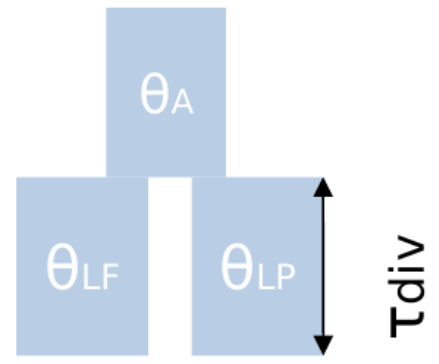

Isolation with Migration - IM

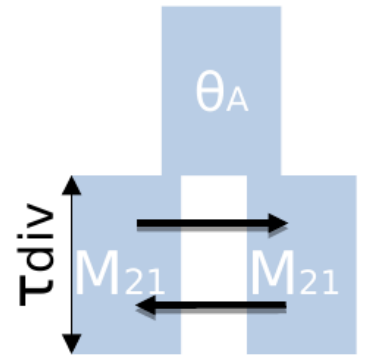

\section{Panmixia - PAN}

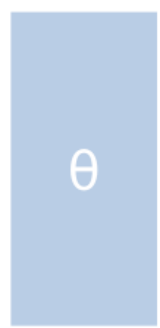

Ancient Migration - AM

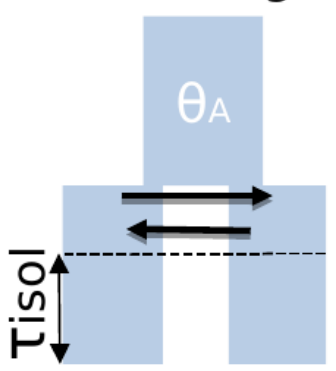

Secondary Contact SC

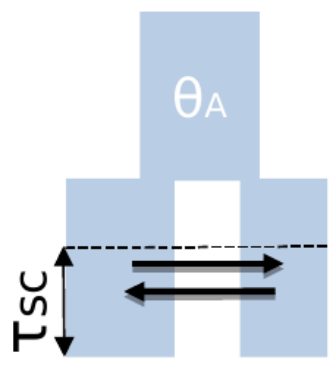


807 Figure 3: Curves of out-of-bag errors rates and Estimation of variable importance.

808 Data based on one random forest, each composed of 1,000 trees obtained from a trained set of 50,000

809 simulated predictor variables (summary statistics). The response variable is the demographic model.

810 Example taken from the Aa river. Estimation for the remaining rivers yielded similar results and are

811 presented in table S2 and Figure S1 

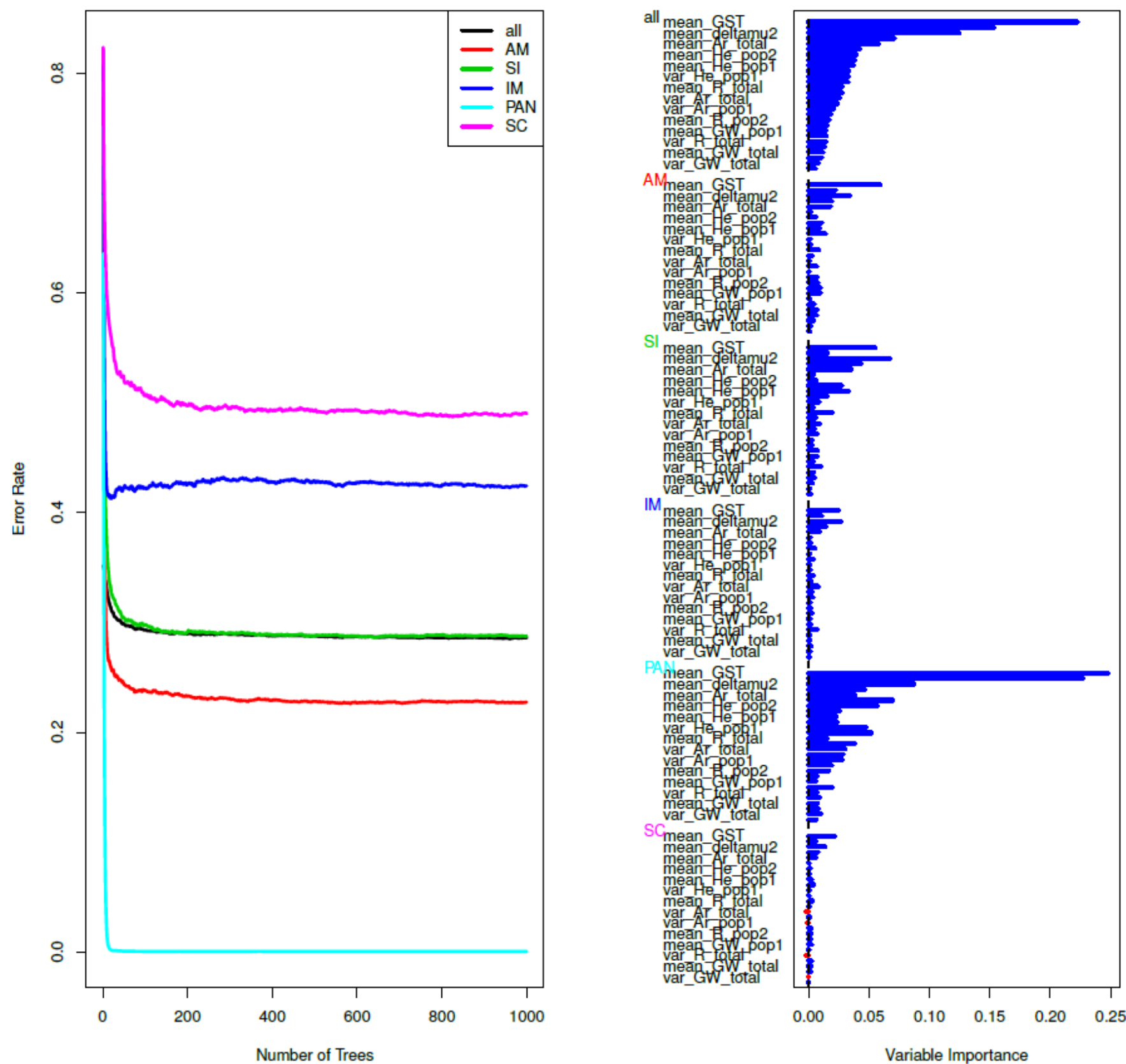